\title{
A randomised trial assessing the acceptability and effectiveness of providing generic versus tailored feedback about health risks for a high need primary care sample
}

\author{
Natasha Noble ${ }^{1 *}$, Christine Paul ${ }^{1}$, Mariko Carey ${ }^{1}$, Stephen Blunden ${ }^{2}$ and Nicole Turner ${ }^{3}$
}

\begin{abstract}
Background: Tailored feedback has been shown to be effective for modifying health risk behaviours and may aid the provision of preventive care by general practitioners (GPs). However, provision of tailored patient feedback for vulnerable or socially disadvantaged groups is not well explored. The aims of this study were to examine the acceptability and effectiveness of providing generic compared to tailored feedback on self-reported health risk behaviours among a high need sample of people attending an Aboriginal Community Controlled Health Service (ACCHS).
\end{abstract}

Methods: Participants attending two ACCHSs in regional New South Wales completed a touch screen health risk survey and received either generic or tailored health risk feedback. Participants were asked to complete an exit survey after their appointment. The exit survey asked about feedback acceptability and effectiveness. Self-reported ease of understanding, relevance and whether the generic versus tailored feedback helped patients talk to their GP was compared using Chi-square analysis; The mean number of survey health risks talked about or for which additional actions were undertaken (such as provision of lifestyle advice or referral) was compared using $t$-tests.

Results: Eighty seven participants (36\% consent rate) completed the exit survey. Tailored feedback was rated as more relevant and was more likely to be shown to the participant's GP than generic feedback. There was no difference in the mean number of health risk topics discussed or number of additional actions taken by the GP by type of feedback.

Conclusions: Tailored and generic feedback showed no difference in effectiveness, and little difference in acceptability, among this socially disadvantaged population. Completing a health risk survey and receiving any type of feedback may have overwhelmed more subtle differences in outcomes between the generic and the tailored feedback. Future work to rigorously evaluate the longer-term effectiveness of the provision of tailored health risk feedback for Aboriginal Australians, as well as other high need groups, is still needed. Trial Registration: Australian New Zealand Clinical Trials Registry ANZCTRN12614001205628. Registered 11 November 2014.

Keywords: Social disadvantage, Aboriginal Australians, Health risk behaviours, Lifestyle risk factors, Feedback, Tailored, Generic

\footnotetext{
* Correspondence: Natasha.Noble@newcastle.edu.au

${ }^{1}$ Priority Research Centre for Health Behaviour and School of Medicine and

Public Health, University of Newcastle, Callaghan, NSW 2308, Australia

Full list of author information is available at the end of the article
} 


\section{Background}

High burden of disease associated with modifiable lifestyle risk behaviours

1 Chronic diseases including cardiovascular disease, cancer and diabetes, are the leading cause of death and morbidity worldwide [1]. Modifiable risk factors such as smoking, poor nutrition, high blood pressure and cholesterol, physical inactivity and excess alcohol, are key contributors to the development of these chronic diseases [2, 3]. The potential to improve population health and reduce the burden of chronic disease through preventing or reducing lifestyle and biomedical risk factors is therefore significant.

\section{High risk groups need assistance to address risk factors}

For a range of cultural and historical reasons, socially disadvantaged groups, including many indigenous populations, tend to show a higher prevalence of lifestyle risk factors compared to the general population [4, 5]. For example, smoking rates among indigenous populations from Australia, New Zealand, Canada, and the United States (US) far exceed those of their non-indigenous counterparts [6]. In Australia, risk factors including smoking, alcohol misuse, physical inactivity and excess weight also show a distinct association with socioeconomic status, with those in the lowest quintile of socioeconomic disadvantage being almost two times more likely to smoke, and 1.7 times more likely not to exercise, than those in the top quintile [7]. Therefore, there is a need to reduce the prevalence of modifiable risk factors for such populations, if inequities in health outcomes are to be addressed.

\section{Primary care as a key setting for prevention and management of modifiable risk factors}

Primary care is generally the front-line for health care in most countries, and is therefore well placed to address patient risk factors [8]. Each health care visit represents a potential opportunity for the delivery of preventive health care services [9]. However, rates of delivery of preventive care in general practice remain low [10]. Data indicate that risk factors such as excess alcohol, smoking and being overweight are not detected by General Practitioners (GPs) for a significant proportion of general practice patients $[8,11,12]$.

Lack of practitioner time is one of the most frequently reported barriers to risk factor detection in the primary care setting [13]. Techniques which enable health care providers to efficiently identify patient risks factors may therefore improve provision of preventive care. Routine screening and delivery of point of care feedback is one technique which may help prompt both health care providers and patients to engage in preventive care. The use of such feedback is well tested in some settings. For example, the provision of tailored health risk feedback to individuals appears to influence change across a range of behaviours including alcohol, smoking and nutrition [14-16]. Similarly, decision support systems which provide recommendations for clinicians have shown benefits for preventive care such as screening, counselling and identification of at risk behaviours [17]. However, the acceptability and effectiveness of providing health risk feedback to socially disadvantaged or vulnerable population groups is not as well explored.

\section{Tailored versus generic feedback}

Feedback can range from simple advice to more intensive or tailored information [14]. Generic feedback contains information which is broadly true for an entire population, such as warnings about alcohol consumption in pregnancy; while tailored feedback is derived from personal information provided by the individual, for example providing information about an individual's level of drinking compared to a reference group or recommended safe level $[14,18]$. Tailored health risk feedback contains less redundant information than generic feedback, and is therefore more likely to be read and remembered by individuals [19]. Evidence also suggests that targeting multiple rather than single health behaviours does not diminish the effectiveness of feedback [20, 21]. The use of pictures that are closely linked to text in health education information has also been found to increase attention and recall, particularly for those with lower literacy levels [22].

\section{Aims of current study}

Aboriginal Community Controlled Health Services (ACCHSs) aim to provide culturally appropriate primary care to Aboriginal people [23], with the majority of those attending being of Aboriginal or Torres Strait Islander origin (74-85\%) [24]. Aboriginal Australians, and potentially also other non-Aboriginal people attending ACCHSs, are an example of a socially disadvantaged group with disproportionately high prevalence of modifiable risk factors [25], for whom the acceptability and effectiveness of provision of health risk feedback has not previously been explored. The aims of the current study were therefore to examine, among patients attending an ACCHS:

a) The acceptability of providing generic or tailored feedback on self-reported health risk behaviours, as assessed by i. reported ease of understanding; ii. perceived relevance of the information; and iii. likelihood of the information helping patients to talk to their GP about any relevant survey risk factors; and

b) The effectiveness of tailored feedback compared to generic feedback, for prompting discussion or action 
on relevant risk factors (such as referral or follow up) during the consultation between the GP and patient.

\section{Methods}

\section{Study design and setting}

People attending two ACCHSs in regional New South Wales (NSW, Australia) were invited to complete an anonymous, cross-sectional health risk survey administered on a touch screen computer. Previous work by the authors confirmed that the touch screen survey was highly acceptable to participants in this setting [26]. Appointment sessions (morning or afternoon) were randomised to either the intervention (tailored feedback) or control (generic feedback) condition using a computer algorithm embedded within software developed for the study. The software was run prior to each session to determine session condition. Approximately equal numbers of sessions were randomised to each condition. Randomisation by session was used to minimise contamination between feedback conditions arising from patients potentially sharing their feedback. In general, morning session patients did not overlap with afternoon patients due to a lunch break between sessions. Study recruitment took place over four months in 2012 and 2013. Ethics approval for the study was granted by the University of Newcastle (reference: H-2011-0153) and the Aboriginal Health and Medical Research Council of NSW (reference: 806/11). The CONSORT 2010 checklist of information to include when reporting a randomised trial for this study is included in Additional file 1.

\section{Participants}

Adults ( $\geq 18$ years) attending the ACCHS for a GP appointment, who were physically and mentally able to provide informed consent and complete the survey, were eligible to participate. Aboriginal and non-Aboriginal people were invited to take part. GPs were informed about the study in person (either via individual [site 1] or group [site 2] meetings) and their consent assumed through agreement of the Chief Executive Officers of the participating services. GPs were shown examples of the two types of feedback but did not receive any specific training regarding responding to the feedback.

\section{Procedure}

Participants were approached by a Research Assistant (RA) in the waiting room and invited to complete a health risk survey while waiting for their appointment. Assistance to complete the survey was offered as required. An Aboriginal RA undertook patient recruitment for half of the recruitment period. Participants were asked to have their weight and height measured (optional), and were able to end the survey if called in for their appointment.
After completing the survey, participants were offered printed generic or tailored feedback (depending on session randomisation) and asked to complete a brief exit survey after their GP appointment. An identification slip given to participants was used to link their health risk and exit survey data. Participants were told that they could show the feedback to their doctor if they wanted, and instructed to ask their doctor or health worker if they had any questions about the feedback. It was not possible to blind participants, health care providers or researchers to allocation to intervention condition. A flow chart showing participant recruitment and allocation is shown in Fig. 1.

\section{Measures}

Health risk survey Details about the health risk survey have been published elsewhere [26]. Briefly, the survey assessed self-reported risk factor status for up to 11 key health risks including: body mass index, smoking status, alcohol consumption, physical activity, fruit and vegetable intake, depression, drug use, and time since last screening for blood pressure, cholesterol, diabetes or HbA1c for those with diabetes, and cancers (including cervical, breast and colorectal cancers, according to participant age and gender). A copy of the text of the health risk survey is available in Additional file 2.

Exit survey Participants were asked to complete an eightitem exit survey presented on a second touchscreen computer immediately after their GP appointment. The exit survey asked participants to self-report any of the 11 health risks that they had talked to their GP about during their appointment (including 'none of these' and 'I prefer not to say' options). Participants were also asked whether the feedback (called the 'checklist') helped them to talk about any of these topics (Yes-I gave the checklist to the doctor/Yes- the checklist gave me some ideas about what to ask the doctor/ No- I didn't use the checklist/ Not sure), and whether any additional actions were taken by their GP (Gave me information (e.g., a website or pamphlet)/ Helped me plan changes to my lifestyle/ Organised a follow up appointment for me/ etc.). For the latter two questions a picture illustrating the health risk topics from the survey was shown and participants were asked to answer just for these topics. Finally, participants were asked to evaluate the feedback by indicating whether it: 'was easy to understand,' 'was relevant to me', and 'will help me improve my health' (Yes/ No/ Not sure). A copy of the text of the exit survey is available in Additional file 3.

\section{Health risk feedback}

Generic feedback included basic lifestyle recommendations and test screening intervals (for those at average risk) based on national guidelines [27-30], for the 11 risk factors 


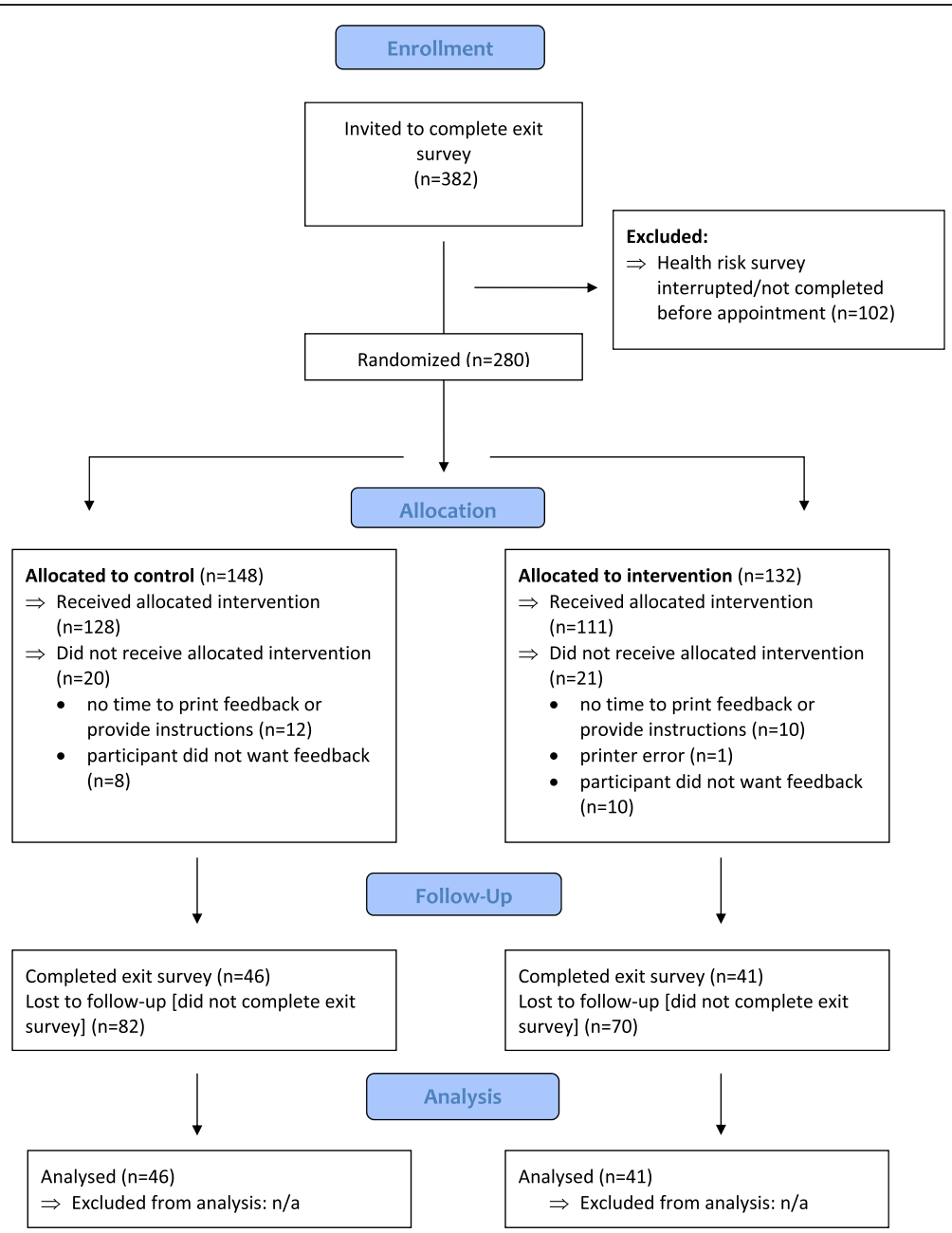

Fig. 1 Flow chart of participant recruitment and allocation

covered in the survey. For example, generic feedback recommendations for smoking were: "If you are a smoker, quitting smoking will improve your health. Talk to your doctor or health worker about ways to quit". Tailored feedback was generated by comparing each participant's survey responses to national guidelines or other accepted cut-offs, and displayed only those factors for which the participant was at risk. Tailored feedback showed the participant's current status compared to the guidelines (e.g., "Your weight $=100 \mathrm{~kg}$. A healthy weight for you $=88 \mathrm{~kg}$ ", calculated using a Body Mass Index of $25 \mathrm{~kg} / \mathrm{m}^{2}$ and participants' measured height), and listed any screening tests for which the participant was overdue. Both types of feedback included simple advice for improving each risk factor, and used pictures, colour and minimal text to maximise appeal [22]. Feedback design and content was based broadly on public health guidelines (e.g., $[30,31])$, as well as input from project collaborators with expertise in Indigenous health and from staff at participating ACCHSs. Examples of the generic and tailored feedback are shown in Additional file 4. At the second site only, a separate 'GP prompt sheet' was added to the tailored feedback for patients, based on recommendations from staff at this service. Participants were instructed that they could give this sheet to their doctor if they wanted. The GP prompt sheet consisted of a separate page showing any health topics that a participant indicated they would like more advice about or help with (see also Additional file 4).

\section{Analysis}

Simple proportions and chi-square tests (or Fisher's exact tests for small cell sizes) were used to assess consent bias and to compare proportions of participants agreeing with statements about the acceptability of the feedback and whether the feedback was useful during their appointment. The mean number of health topics discussed and mean number of actions undertaken for those who received the generic versus the tailored feedback were compared using $t$ tests. Due to the low response rate (see below), results for the tailored feedback at both sites (with and without the 
additional GP prompt sheet) were combined and analysed as a single intervention condition.

\section{Results \\ Sample}

The overall consent rate for the health risk survey was $69 \%$. There were no significant differences between the age and gender of consenters and non-consenters (p's > .05; data not shown). Non-Aboriginal people were significantly more likely to consent, compared to the proportions of total active Aboriginal and non-Aboriginal patients registered in the clinical records of the ACCHSs, $x^{2}$ $(1, N=4091)=9.71, p=0.002$. Demographic data confirmed that the total sample represented a broadly socially and economically disadvantaged group, with $66 \%$ reporting Centrelink (government welfare) as their source of income, and the majority of the sample (56\%) having a highest education level of year 10 or below, compared to approximately $35 \%$ of the general Australian population [32] (data not shown).

Of the 239 participants who were given the feedback, 87 completed the exit survey ( $36 \%$ consent rate). Of these, 46 participants (53\%) received the generic and 41 participants the tailored feedback. The demographic characteristics of those who completed the exit survey are shown in Table 1 . There were no significant differences between participants who did and did not complete the exit survey in terms of age, gender or Aboriginal status (all p's $>.05$, data not shown).

\section{Patient assessment of the generic and tailored feedback}

The percentage of participants agreeing with statements assessing the two types of feedback (i.e., those responding 'yes') are shown in Fig. 2. Participants were significantly more likely to agree that the tailored feedback was 'relevant to me' compared to the generic feedback, Fisher's exact: $\chi^{2}(1, N=87)=5.22, p=.03$, while agreement did not differ for the other statements ( $p s>.05)$.

\section{Usefulness of the feedback}

For those who reported talking to their GP about any of the health risks in the survey $(n=38$ generic, $n=32$ tailored), participants were asked whether the feedback checklist helped them during their appointment. Responses are shown in Table 2 . Significantly more participants who received the tailored feedback reported showing this to their GP than those who received the generic feedback, Fisher's exact $\chi^{2}(2, N=70)=7.30, p=.03$.

\section{Effectiveness of generic versus tailored feedback}

The average number of survey health topics talked about during the appointment was $2.85(\mathrm{SD}=2.33)$. The majority of participants $(82 \%)$ reported talking to their GP about at least one of the survey health risks in their
Table 1 Demographic characteristics of participants completing the exit survey by group allocation $(n=87)$

\begin{tabular}{|c|c|c|c|}
\hline \multirow[t]{2}{*}{ Demographics } & $\begin{array}{l}\text { Generic feedback } \\
(n=46)\end{array}$ & $\begin{array}{l}\text { Tailored feedback } \\
(n=41)\end{array}$ & $\begin{array}{l}\text { All exit survey } \\
\text { completers }\end{array}$ \\
\hline & n (\%) & n (\%) & n (\%) \\
\hline \multicolumn{4}{|l|}{ Gender } \\
\hline Male & 17 (37 \%) & 18 (44 \%) & $35(40 \%)$ \\
\hline Female & 29 (63 \%) & $23(56 \%)$ & $52(60 \%)$ \\
\hline \multicolumn{4}{|l|}{ Age } \\
\hline$<35 \mathrm{yrs}$ & 15 (33 \%) & $11(27 \%)$ & $26(30 \%)$ \\
\hline $35-54$ yrs & 17 (37 \%) & 15 (37 \%) & $32(37 \%)$ \\
\hline$\geq 55$ yrs & 14 (30 \%) & 15 (37 \%) & $29(33 \%)$ \\
\hline \multicolumn{4}{|l|}{ Aboriginal status } \\
\hline Aboriginal & 34 (74 \%) & 28 (68 \%) & 62 (71 \%) \\
\hline Non-Aboriginal & 12 (26 \%) & $13(32 \%)$ & $25(29 \%)$ \\
\hline \multicolumn{4}{|l|}{$\begin{array}{l}\text { Highest level of } \\
\text { education }^{\mathrm{a}}\end{array}$} \\
\hline Yr 10 or below & 25 (54 \%) & $25(61 \%)$ & $50(58 \%)$ \\
\hline Yr 12 & $6(13 \%)$ & $3(7 \%)$ & $9(10 \%)$ \\
\hline TAFE/Other & $6(13 \%)$ & $6(15 \%)$ & $12(14 \%)$ \\
\hline $\begin{array}{l}\text { University/ } \\
\text { Tertiary }\end{array}$ & 9 (20\%) & $6(15 \%)$ & 15 (17 \%) \\
\hline \multicolumn{4}{|l|}{$\begin{array}{l}\text { Employment } \\
\text { status }\end{array}$} \\
\hline Employed & 15 (33 \%) & 17 (42 \%) & 32 (37 \%) \\
\hline $\begin{array}{l}\text { Unemployed/ } \\
\text { supported }\end{array}$ & 31 (67 \%) & 24 (59 \%) & 55 (63 \%) \\
\hline
\end{tabular}

appointment, regardless of whether or not they reported using the feedback. The mean number of topics discussed did not differ between those who received the generic $(M=2.87, S D=2.39)$ versus the tailored feedback $(M=2.83, S D=2.28 ; t(85)=0.08, p=.94)$. There was also no difference in the average number of additional actions related to survey health risks taken by the GP (such as help plan changes to lifestyle, gave me information, organised a follow up appointment) between participants who received the generic versus tailored feedback $(t(85)=0.85, p=.40)$.

\section{Discussion}

Tailored feedback was rated as 'more relevant', and was more likely to be shown to the GP than the generic feedback. Given that the tailored feedback included personalised risk factor information, it is not surprising that it was rated as more relevant. Almost two-thirds of participants given the tailored feedback indicated that they either showed the feedback to their GP or it gave them some ideas about what to ask the doctor, compared to less than half of those who received generic feedback. Anecdotally, the tailored feedback prompted a greater 


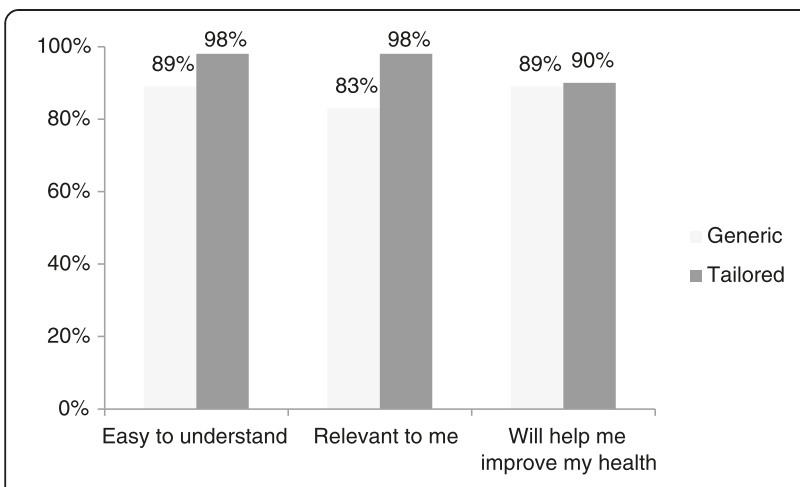

Fig. 2 Assessment of the generic and tailored feedback (\% who responded 'yes' to acceptability statements; $n=87$ )

response from participants than the generic feedback, including several who were surprised by their recommended healthy weight, and one participant who commented that seeing her depression score prompted her to bring this up with her GP. However, participants did not rate the tailored feedback as any more likely to help them improve their health, or any easier to understand, than the generic feedback.

The type of feedback did not appear to influence interaction with the GP in terms of the number of health risks talked about or other actions offered. An average of almost three health survey topics was discussed for all participants completing the exit survey regardless of type of feedback given, and an average of $36 \%$ of these participants indicated that their GP helped them to plan changes to their lifestyle during their appointment (data not shown). It is likely that GPs within the ACCHS setting are already discussing relevant health risks with their patients, as indicated by the majority of participants who reported discussing at least one survey health risk, independently of whether they showed the feedback to their GP. Alternatively, it may be that the process of screening by completing the health risk survey (as suggested by McPhail et al. 2014 [33]), and being provided with feedback, prompted discussion, even if the feedback was not shown to the GP and/or the participant did not report using the feedback.

Table 2 Proportion of participants using the feedback to talk to their GP about survey health risks

\begin{tabular}{lll}
\hline Use of feedback during GP appointment & $\begin{array}{l}\text { Generic } \\
\text { feedback } \\
\mathrm{n}(\%)\end{array}$ & $\begin{array}{l}\text { Tailored } \\
\text { feedback } \\
\mathrm{n}(\%)\end{array}$ \\
\hline I gave/showed the feedback to the doctor & $2(5 \%)$ & $9(28 \%)$ \\
The feedback gave me some ideas about & $14(37 \%)$ & $11(34 \%)$ \\
what to ask the doctor & $22(58 \%)$ & $12(38 \%)$ \\
\hline I didn't use the checklist/not sure & & \\
\hline
\end{tabular}

In contrast to previous findings such as those reported by de Vries et al. [34], and Skinner et al. [16], tailored feedback did not outperform generic feedback in this study. In a meta-analysis of tailored health behaviour change materials, tailored interventions with more than one contact with participants (for example, providing three feedback letters at different times [34]) had significantly larger effect sizes than those with only one point of contact [18]. Also, materials which were tailored to multiple concepts such as participants' stage of change, and/or self-efficacy, as well as behaviour, were more effective than those tailored on behaviour alone [18]. Thus a more intensive approach to the provision of tailored feedback, such as one tailored to stage of change, or to patient and/or health practitioner perception of risk [35], in addition to behaviour, and using an outcome measure not limited to immediate interaction between the participant and their GP, may have revealed a differential effectiveness of the two types of feedback for people attending an ACCHS. Alternatively, it may be that providing generic feedback to participants provides enough of a trigger to prompt discussion with the GP in this setting.

There were a number of limitations to this study. Firstly, the poor overall response rate and small sample who completed the exit survey substantially limit the power of the study to detect any differences between feedback types. Despite the exit survey being as brief as possible, the majority of participants indicated when invited that they would not have time to complete the exit survey after their appointment. Participants attending the ACCHSs often waited for a long time, came with others, and/or had others waiting for them to finish their appointment. In this setting, an exit survey may not be an effective way to obtain post-appointment data. Alternatively, some kind of incentive may need to be offered to encourage participation. It is likely that those who completed the exit survey had a greater interest in their health, and therefore the results may over-report the usefulness of both the generic and tailored feedback. An objective measure of the interaction between patient and GP (e.g., audio-recording) would also have allowed more systematic identification of relevant health risk discussions (which was limited to any relevant risk a patient reported talking to their doctor about, regardless of the nature or depth of the discussion) or actions. Secondly, there were inconsistencies in providing feedback and inviting participants to complete the exit survey, due to time constraints, participants not wanting feedback, and incomplete surveys (for which tailored feedback could not be generated). These inconsistencies also limit the generalizability of the results as not all eligible participants were invited to complete an exit survey and provide data about the feedback. Lastly, a key limitation 
involves possible contamination between feedback conditions based on morning or afternoon appointment sessions. As the same practitioners saw patients presenting with both types of feedback, it is possible that advice or action in response to one type of feedback spilled over to patients receiving the other. However, attempting to randomise feedback on another basis would have introduced additional sources of bias such those arising from differences between practitioners or practices.

\section{Conclusions}

Both generic and tailored feedback on multiple risk behaviours appeared to be largely acceptable to this sample of people attending an ACCHS, who are broadly representative of a population experiencing social and economic disadvantage. Tailored feedback was no more effective in prompting health risk discussion or action between participants and their GP than generic feedback. Almost $90 \%$ of participants agreed that either type of feedback would help them improve their health. It is likely that the impact of completing the health risk survey, together with receiving some form of feedback, overwhelmed more subtle differences in outcomes between the generic and the tailored feedback, especially given the small sample size and limited outcome measures. Future work to rigorously evaluate acceptability and longerterm effectiveness of the provision of tailored health risk feedback for Aboriginal Australians, as well as other high need groups, is still needed. Future work may also consider tailoring of feedback to additional concepts such as stage of change or self-efficacy as well as behaviour, and exploring the impact of providing repeated feedback contacts, in assessing feedback effectiveness.

\section{Additional files}

Additional file 1: CONSORT 2010 checklist for randomised trials.

Additional file 2: Health risk survey (text version).

Additional file 3: Exit survey (text version).

Additional file 4: Example of the generic and tailored feedback.

\section{Abbreviations}

ACCHS: Aboriginal Community Controlled Health Service; GP: General Practitioner; NSW: New South Wales.

Competing interests

The author(s) declare that they have no competing interests.

\section{Authors' contributions}

NN developed the study materials, conducted data collection, undertook analysis and interpretation of the data, and drafted the manuscript. CP made substantial contributions to the study conception and design, provided support during data collection, and assisted with drafting and revision of the manuscript. MC made substantial contributions to the study design and development of feedback materials, and assisted with drafting and revision of the manuscript. SB and NT provided feedback on study design and specific study materials, facilitated and provided assistance with data collection, and provided critical feedback and revision of the manuscript. All authors have given final approval for this version of the manuscript to be published.

\section{Acknowledgments}

Sincere thanks to all the staff (in particular the CEOs, Leanne Dryden, Jill McDonald, Steve Terrey, Annette Olive, and reception staff) who supported this research, and to the participants who kindly completed the survey and made this study possible. Many thanks also to Dr Mark Wallis for development of the feedback software and ongoing software support, and to Professor Katherine Conigrave, Dr Kylie Lee, Ms Jessica Stewart and L/Professor Robert Sanson-Fisher for their valuable input into the study design and materials. This manuscript was supported by a Strategic Research Partnership Grant from Cancer Council NSW to the Newcastle Cancer Control Collaborative, and a NSW Health Drug and Alcohol Research Grant. A/Prof Christine Paul is supported by an NHMRC Career Development Fellowship. Infrastructure support was provided by the Hunter Medical Research Institute. These funding bodies had no involvement in the conduct of the research or the preparation of this manuscript.

\section{Author details}

'Priority Research Centre for Health Behaviour and School of Medicine and Public Health, University of Newcastle, Callaghan, NSW 2308, Australia. ${ }^{2}$ Casino Aboriginal Medical Service, Casino, NSW 2470, Australia. ${ }^{3}$ School of Medicine and Public Health and Department of Rural Health, University of Newcastle, Callaghan, NSW 2308, Australia.

Received: 25 September 2014 Accepted: 22 July 2015

Published online: 05 August 2015

\section{References}

1. Yach D, Hawkes C, Gould CL, Hofman KJ. The global burden of chronic diseases: overcoming impediments to prevention and control. JAMA. 2004;291(21):2616-22.

2. Lim SS, Vos T, Flaxman AD, Danaei G, Shibuya K, Adair-Rohani H, et al. A comparative risk assessment of burden of disease and injury attributable to 67 risk factors and risk factor clusters in 21 regions, 1990-2010: a systematic analysis for the Global Burden of Disease Study 2010. Lancet. 2013;380(9859):2224-60.

3. Lopez AD, Mathers CD, Ezzati M, Jamison DT, Murray CJ. Global and regional burden of disease and risk factors, 2001: systematic analysis of population health data. The Lancet. 2006;367(9524):1747-57.

4. Gracey M, King M. Indigenous health part 1: determinants and disease patterns. The Lancet. 2009;374(9683):65-75.

5. Pampel FC, Krueger PM, Denney JT. Socioeconomic disparities in health behaviors. Ann Rev Sociol. 2010;36:349-70.

6. DiGiacomo M, Davidson PM, Abbott PA, Davison J, Moore L, Thompson SC. Smoking cessation in Indigenous populations of Australia, New Zealand, Canada, and the United States: elements of effective interventions. Int J Environ Res Public Health. 2011;8(2):388-410.

7. Glover JD, Hetzel DM, Tennant SK. The socioeconomic gradient and chronic illness and associated risk factors in Australia. Australia New Zealand Health Policy. 2004; 1 (1):8-16.

8. Yoong SL, Carey ML, Sanson-Fisher RW, D'Este CA, Mackenzie L, Boyes A. A cross-sectional study examining Australian general practitioners' identification of overweight and obese patients. J Gen Intern Med. 2014;29(2):328-34

9. Balas EA, Weingarten $S$, Garb $C T$, Blumenthal D, Boren $S A$, Brown GD. Improving preventive care by prompting physicians. Arch Intern Med. 2000;160(3):301-8

10. Yarnall KS, Pollak KI, Østbye T, Krause KM, Michener JL. Primary care: is there enough time for prevention? Am J Public Health. 2003;93(4):635-41.

11. Dickinson JA, Wiggers J, Leeder SR, Sanson-Fisher RW. General practitioners' detection of patients' smoking status. Med J Aust. 1989;150(8):420-2.

12. Paul C, Yoong SL, Sanson-Fisher R, Carey M, Russell G, Makeham M. Under the radar: a cross-sectional study of the challenge of identifying at-risk alcohol consumption in the general practice setting. BMC Fam Pract. 2014;15(1):74-81

13. Dennis S, Williams A, Taggart J, Newall A, Denney-Wilson E, Zwar N, et al. Which providers can bridge the health literacy gap in lifestyle risk factor 
modification education: a systematic review and narrative synthesis. BMC Fam Pract. 2012;13(1):44-73.

14. DiClemente CC, Marinilli AS, Singh M, Bellino LE. The role of feedback in the process of health behavior change. Am J Health Behav. 2001;25(3):217-27.

15. Kreuter MW, Oswald DL, Bull FC, Clark EM. Are tailored health education materials always more effective than non-tailored materials? Health Educ Res. 2000;15(3):305-15.

16. Skinner CS, Campbell MK, Rimer BK, Curry S, Prochaska JO. How effective is tailored print communication? Ann Behav Med. 1999;2(4):290-8.

17. Garg AX, Adhikari NK, McDonald H, Rosas-Arellano MP, Devereaux PJ, Beyene J, et al. Effects of computerized clinical decision support systems on practitioner performance and patient outcomes: a systematic review. JAMA. 2005:293(10):1223-38.

18. Noar SM, Benac CN, Harris MS. Does tailoring matter? Meta-analytic review of tailored print health behavior change interventions. Psychol Bull. 2007;133(4):673-93.

19. De Vries H, Brug J. Computer-tailored interventions motivating people to adopt health promoting behaviours: introduction to a new approach. Patient Educ Counsel. 1999;36(2):99-105.

20. Smeets T, Kremers S, De Vries H, Brug J. Effects of tailored feedback on multiple health behaviors. Ann Behav Med. 2007;33(2):117-23.

21. Vandelanotte C, De Bourdeaudhuij I, Sallis JF, Spittaels H, Brug J. Efficacy of sequential or simultaneous interactive computer-tailored interventions for increasing physical activity and decreasing fat intake. Ann Behav Med. 2005;29(2):138-46.

22. Houts PS, Doak CC, Doak LG, Loscalzo MJ. The role of pictures in improving health communication: a review of research on attention, comprehension, recall, and adherence. Patient Educ Couns. 2006:61(2):173-90.

23. Definitions. [http://www.naccho.org.au/aboriginal-health/definitions/]

24. Australian Institute of Health and Welfare. Healthy for Life- Aboriginal Community Controlled Health Services: Report Card. Cat. no. IHW 97. Canberra: AlHW; 2013.

25. Vos T, Barker B, Begg S, Stanley L, Lopez AD. Burden of disease and injury in Aboriginal and Torres Strait Islander Peoples: the Indigenous health gap. Int J Epidemiol. 2009;38(2):470-7.

26. Noble NE, Paul CL, Carey ML, Sanson-Fisher RW, Blunden SV, Stewart JM, et al. A cross-sectional survey assessing the acceptability and feasibility of selfreport electronic data collection about health risks from patients attending an Aboriginal Community Controlled Health Service. BMC Med Inform Decis Mak. 2014;14(1):34

27. Department of Health and Aging. Physical activity guidelines for adults. 2005.

28. NACCHO, RACGP. National guide to a preventive health assessment for Aboriginal and Torres Strait Islander people. 2nd ed. South Melbourne: The RACGP; 2012.

29. NHMRC \& DOHA. Dietary guidelines for Australian adults: A guide to healthy eating. Canberra: NHMRC; 2005.

30. Royal Australian College of General Practitioners. Guidelines for Preventive Activities in General Practice. 7th ed. 2009.

31. Royal Australian College of General Practitioners National Standing Committee. Smoking, Nutrition, Alcohol and Physical activity (SNAP). In: Ageing AGDoHa, editor. A population health guide to behavioural risk factors in general practice. South Melbourne: Royal Australian College of General Practitioners; 2004.

32. Education. 2076.0 - Census of Population and Housing: Characteristics of Aboriginal and Torres Strait Islander Australians, 2011. [http://www.abs.gov.au/ ausstats/abs@.nsf/Lookup/2076.0main+features302011]

33. McPhail S, Schippers M. An evolving perspective on physical activity counselling by medical professionals. BMC Fam Pract. 2012;13(1):31-9.

34. De Vries H, Kremers SPJ, Smeets T, Brug J, Eijmael K. The effectiveness of tailored feedback and action plans in an intervention addressing multiple health behaviors. Am J Health Promot. 2008;22(6):417-25.

35. Bonner C, Jansen J, McKinn S, Irwig L, Doust J, Glasziou P, et al. Communicating cardiovascular disease risk: An interview study of general practitioners' use of absolute risk within tailored communication strategies BMC Fam Pract. 2014;15(1):106-14.

\section{Submit your next manuscript to BioMed Central and take full advantage of:}

- Convenient online submission

- Thorough peer review

- No space constraints or color figure charges

- Immediate publication on acceptance

- Inclusion in PubMed, CAS, Scopus and Google Scholar

- Research which is freely available for redistribution

Submit your manuscript at www.biomedcentral.com/submit 